Trinity University

Digital Commons@ Trinity

Mathematics Faculty Research

Mathematics Department

$11-2014$

\title{
A Combinatorial Proof of a Theorem of Katsuura
}

Brian K. Miceli

Trinity University, bmiceli@trinity.edu

Follow this and additional works at: https://digitalcommons.trinity.edu/math_faculty

Part of the Mathematics Commons

\section{Repository Citation}

Miceli, B.K. (2014). A combinatorial proof of a theorem of Katsuura. The College Mathematics Journal, 45(5), 365-369. doi:10.4169/ college.math.j.45.5.365.

This Article is brought to you for free and open access by the Mathematics Department at Digital Commons @ Trinity. It has been accepted for inclusion in Mathematics Faculty Research by an authorized administrator of Digital Commons @ Trinity. For more information, please contact jcostanz@trinity.edu. 


\section{A Combinatorial Proof of a Theorem of Katsuura}

Brian K. Miceli

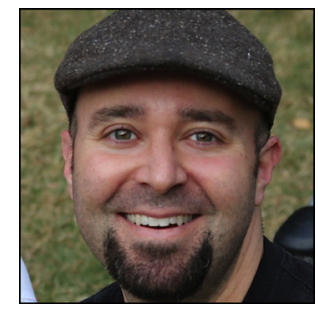

Brian Miceli (bmiceli@trinity.edu) is an associate professor of mathematics at Trinity University in San Antonio, Texas.

He received his B.S. from California Polytechnic State University, San Luis Obispo, and his Ph.D. from the University of California, San Diego. Including his home town, he has always lived in a city beginning with "San" that lies southeast of his previous city of residence, leaving San Juan, Puerto Rico as his most likely retirement destination. He enjoys rooting for the 49ers, cooking, cruising the zoo with his wife and daughter, going to rock concerts, and playing racquetball.

In mathematics, one does not have to look too hard to find a plethora of problems asking to show that $A=B$. A combinatorial (or counting) proof of such a formula would be one where we show, in two separate ways, that both sides of our formula enumerate the same set of objects. Indeed, when one sees a beautiful formula involving binomial coefficients, integral powers, and factorials, a combinatorial proof surely seems plausible. Such a formula was provided by Dence [2], who in working with Laplace transforms and row-sums on Pascal's triangle, provided two conjectures that can be combined into the following statement.

Theorem 1. Suppose $k, n$ are positive integers with the same parity such that $k \leq n$. Then

$$
\sum_{j=0}^{\left\lfloor\frac{n-1}{2}\right\rfloor}(-1)^{j}\left(\begin{array}{l}
n \\
j
\end{array}\right)(n-2 j)^{k}= \begin{cases}0 & \text { if } k<n, \text { and } \\
2^{n-1} n ! & \text { if } k=n\end{cases}
$$

Dence's conjecture was first proved in 2009 as consequence of following result.

Theorem 2 (Katsuura [4]). Suppose $k, n$ are positive integers such that $k \leq n$ and $x, y \in \mathbb{C}$. Then

$$
\sum_{j=0}^{n}(-1)^{j}\left(\begin{array}{l}
n \\
j
\end{array}\right)(y-x j)^{k}= \begin{cases}0 & \text { if } k<n, \text { and } \\
x^{n} n ! & \text { if } k=n .\end{cases}
$$

Later, using identities among arbitrary complex polynomials, Fengming, Kim, and Yeong [3] proved a more general theorem from which Katsuura's result, and hence Theorem 1, are deduced as special cases. As Katsuura remarks in [4], Theorem 2 is

http://dx.doi.org/10.4169/college.math.j.45.5.365

MSC: 05A19, 05A05 
"curious" and "almost too good to be true since it says that the left hand side of the equation is independent of the choice of the number $y$. ." The goal of this paper is to give a combinatorial proof of Theorem 2, and we hope that such a proof will shed some light as to why the choice of $y$ does not affect this sum. However, with the $(-1)^{j}$ term and the fact that $x$ and $y$ need not be positive (or even integers!), we need to be a little more clever about how to use our combinatorial toolbox. As it turns out, our proof will involve both the involution principle (IP) applied to certain sets of colored words and the fundamental theorem of algebra.

For the full details of the IP method, we refer the reader to Benjamin and Quinn [1], but we give a quick review here. An involution is a bijective function $g: X \rightarrow X$ such that $g(g(x))=x$ for every $x \in X$. Any $x \in X$ such that $g(x)=x$ is called a fixed point of the involution, and we see that our involution partitions $X$ into the two sets, $g_{\text {fix }}(X)=\{x \in X \mid g(x)=x\}$ and $g_{\text {n-fix }}(X)=\{x \in X \mid g(x) \neq x\}$.

Now, suppose our goal is to compute the sum $\mathcal{S}(A)=\sum_{y \in A} \gamma(y)$ where $A$ is a set of objects and $\gamma$ represents a weight function on the objects of $A$. We would like to find an involution $f: A \rightarrow A$ such that $\gamma(y)=-\gamma(f(y))$ whenever $y \in f_{\mathrm{n} \text {-fix }}(A)$. Here, $f$ is called a sign-reversing involution, and given such an $f$, we rewrite our desired sum as

$$
\mathcal{S}(A)=\sum_{y \in f_{\mathrm{fix}}(A)} \gamma(y)+\sum_{y \in f_{\mathrm{n}-\mathrm{fix}}(A)} \gamma(y) .
$$

Because $f$ is sign-reversing, the weights in the right sum come in pairs that cancel out. Accordingly, the sum on the right is 0 , leaving

$$
\mathcal{S}(A)=\sum_{y \in f_{\mathrm{fix}}(A)} \gamma(y) .
$$

We can then use this formula for computing $\mathcal{S}(A)$, hoping that it is an easier task to sum over just the fixed points of our involution.

For our combinatorial proof of Theorem 2, we will be interested in counting colored words over a certain alphabet. Given a finite set $W=\left\{w_{1}, \ldots, w_{s}\right\}$, we define a word over $W$ to be any string consisting of elements (called letters) of $W$ and we let $W^{*}$ denote the set of all words over $W$. The number of letters of $w$ is called the length of $w$, denoted by $|w|$. For example, if $B=\{0,1\}$, then $b=0010110 \in B^{*}$ and $|b|=7$. We say that $w \in W^{*}$ is a permutation of $W$ if $|w|=s$ and each $w_{i}$ appears exactly once in $w$. Given a set of colors $\mathcal{C}=\left\{c_{1}, \ldots, c_{m}\right\}$, we define $W_{\mathcal{C}}$ to be the set of elements of $W$ where each element is colored with the colors of $\mathcal{C}$, i.e., $W_{\mathcal{C}}$ contains $m$ distinct copies of each element of $W$. We then define a $\mathcal{C}$-colored word to be any element of $W_{\mathcal{C}}^{*}$. Here, $u^{v}$ denotes that the letter $u$ is colored with the color $v$. For example, if $\mathcal{C}=\{1,2,3\}$, then $B_{\mathcal{C}}=\left\{0^{1}, 0^{2}, 0^{3}, 1^{1}, 1^{2}, 1^{3}\right\}$ and $d=0^{1} 0^{3} 1^{1} 0^{1} 1^{2} 1^{2} 0^{3} \in B_{\mathcal{C}}^{*}$ with $|d|=7$.

Before we prove Theorem 2, we prove the same result for the case that $x$ and $y$ are positive integers rather than arbitrary complex numbers.

Theorem 3. Suppose $x, y, k, n$ are positive integers such that $k \leq n$. Then

$$
\sum_{j=0}^{n}(-1)^{j}\left(\begin{array}{l}
n \\
j
\end{array}\right)(y-x j)^{k}= \begin{cases}0 & \text { if } k<n, \text { and } \\
x^{n} n ! & \text { if } k=n .\end{cases}
$$


Proof. Let $Y=\left\{b_{1}, \ldots, b_{y}\right\}, \mathcal{C}=\{1, \ldots, x\}$, and $[n]=\{1, \ldots, n\}$. Define

$$
A=\left\{(T, w) \mid T \subseteq[n] \text { and } w \in\left(Y \cup T_{\mathcal{C}}\right)^{*} \text { with }|w|=k\right\} .
$$

For example, if $n=10, k=8, x=3$, and $y=3$, then

$$
\left(\{1,2,6,9,10\}, b_{2} 2^{1} 6^{3} b_{3} b_{1} 1^{3} 9^{2} b_{1}\right) \in A .
$$

For any $(T, w) \in A$, let the weight of $(T, w)$ be $\gamma((T, w))=(-1)^{|T|+u(w)}$ where $u(w)$ is the number of letters in $w$ that come from $T_{\mathcal{C}}$. Using the example above, $T=\{1,2,6,9,10\}$, so $|T|=5$, and the word $w=b_{2} 2^{1} 6^{3} b_{3} b_{1} 1^{3} 9^{2} b_{1}$ contains four letters from $T_{\mathcal{C}}$, therefore

$$
\gamma\left(\left(\{1,2,6,9,10\}, b_{2} 2^{1} 6^{3} b_{3} b_{1} 1^{3} 9^{2} b_{1}\right)\right)=(-1)^{5+4}=-1 .
$$

For any fixed $j$, if $|T|=j$, then there are $\left(\begin{array}{l}n \\ j\end{array}\right)$ such $j$-element subsets of $[n]$ and there are $(y+x j)^{k}$ words of length $k$ consisting of letters from $Y \cup T_{\mathcal{C}}$. Thus, the number of pairs $(T, w) \in A$ such that $|T|=j$ is $\left(\begin{array}{c}n \\ j\end{array}\right)(y+x j)^{k}$. Replacing $x j$ with $-x j$ gives each pair $(T, w)$ a weight of $(-1)^{u(w)}$ and, accordingly,

$$
(-1)^{j}\left(\begin{array}{l}
n \\
j
\end{array}\right)(y-x j)^{k}=\sum_{\substack{(T, w) \in A \\
|T|=j}} \gamma((T, w))
$$

Summing both side of this equation over all possible values of $j$ gives

$$
\sum_{j=0}^{n}(-1)^{j}\left(\begin{array}{l}
n \\
j
\end{array}\right)(y-x j)^{k}=\sum_{j=0}^{n} \sum_{\substack{(T, w) \in A \\
|T|=j}} \gamma((T, w))=\sum_{(T, w) \in A} \gamma((T, w)) .
$$

Given $(T, w) \in A$, let $\ell$ be the smallest element of $[n]$ for which no colored version appears in $w$, and define $f: A \rightarrow A$ in the following way.

i. If $\ell \in T$, then $f((T, w))=\left(T^{\prime}, w\right)$, where $T^{\prime}=T-\{\ell\}$.

ii. If $\ell \notin T$, then $f((T, w))=\left(T^{\prime}, w\right)$, where $T^{\prime}=T \cup\{\ell\}$.

iii. If no such $\ell$ exists, then $f((T, w))=(T, w)$.

Using our previous example, $\ell=3$ and, since $3 \notin T=\{1,2,6,9,10\}$, step ii above gives

$$
f\left(\left(\{1,2,6,9,10\}, b_{2} 2^{1} 6^{3} b_{3} b_{1} 1^{3} 9^{2} b_{1}\right)\right)=\left(\{1,2,3,6,9,10\}, b_{2} 2^{1} 6^{3} b_{3} b_{1} 1^{3} 9^{2} b_{1}\right) .
$$

This function $f$ is a sign-reversing involution since, if $f((T, w))=\left(T^{\prime}, w\right)$ with $T^{\prime} \neq$ $T$, then $f\left(\left(T^{\prime}, w\right)\right)=(T, w)$ and $\left|T^{\prime}\right|=|T| \pm 1$. Using the IP to compute (1) gives

$$
\sum_{j=0}^{n}(-1)^{j}\left(\begin{array}{l}
n \\
j
\end{array}\right)(y-x j)^{k}=\sum_{f_{\mathrm{fix}}(A)} \gamma((T, w)) .
$$

Given $(T, w) \in f_{\text {fix }}(A)$, it must be the case that $w$ contains some colored version of every element of $[n]$. Since $|w|=k$, there are no fixed points of $f$ in the case that 
$k<n$ and the right-hand side of (2) is 0 . Otherwise $k=n$. In this case, $T$ must be the set $[n]$ in order for $w$ to contain some colored version of every element of $[n]$ and

$$
f_{\text {fix }}(A)=\{([n], w) \mid w \text { is a } \mathcal{C} \text {-colored permutation of }[n]\} .
$$

Now if $([n], w) \in f_{\text {fix }}(A)$, then $\gamma\left(([n], w)=(-1)^{|[n]|+u(w)}=(-1)^{n+n}=1\right.$ and $(2)$ reduces to

$$
\sum_{j=0}^{n}(-1)^{j}\left(\begin{array}{l}
n \\
j
\end{array}\right)(y-x j)^{k}=\sum_{f_{\text {fix }}(A)} 1=\left|f_{\text {fix }}(A)\right| .
$$

Since there are $n$ ! permutations of $[n]$ and $x^{n}$ ways to color each permutation, $\left|f_{\text {fix }}(A)\right|=x^{n} n !$, completing the proof.

From this proof, we see that the number $y$ in our sum corresponds to a set of $y$ letters used to make words that, when we apply our sign-reversing involution $f$, only appear in the nonfixed points of $f$ and thus do not contribute to the final value of the sum.

To show that Theorem 3 extends to any $x, y \in \mathbb{C}$, we use the fundamental theorem of algebra, usually stated as follows.

Theorem 4 (Fundamental theorem of algebra). Let $n$ be a positive integer and suppose that $a(x)=a_{n} x^{n}+a_{n-1} x^{n-1}+\cdots+a_{1} x+a_{0}$ such that each $a_{i} \in \mathbb{C}$ and $a_{n} \neq 0$. Then $a(x)$ has exactly $n$ complex roots, counted with multiplicity.

A consequence of this theorem is that if $p(x)$ is a complex polynomial of finite degree with an infinite number of roots, then it must be the zero polynomial.

Proof of Theorem 2. First let $n, k, y$ be fixed positive integers and define

$$
q_{k}(x)=\sum_{j=0}^{n}(-1)^{j}\left(\begin{array}{l}
n \\
j
\end{array}\right)(y-x j)^{k} .
$$

By Theorem 3, $q_{n}(x)=x^{n} n$ ! for infinitely many values of $x$ and thus the polynomial $p(x)=q_{n}(x)-x^{n} n$ ! has an infinite number of distinct complex roots (the positive integers). However, $p(x)$ is a polynomial of degree at most $n$ and, by the fundamental theorem of algebra, $p(x)=0$ for all $x \in \mathbb{C}$. A similar argument holds in the case where $k<n$, as $q_{k}(x)$ is a polynomial of degree $k$ with infinitely many roots.

Now letting $n, k$ be positive integers and fixing $x \in \mathbb{C}$, a similar argument shows that for every $y \in \mathbb{C}$,

$$
\sum_{j=0}^{n}(-1)^{j}\left(\begin{array}{l}
n \\
j
\end{array}\right)(y-x j)^{k}= \begin{cases}0 & \text { if } k<n, \text { and } \\
x^{n} n ! & \text { if } k=n .\end{cases}
$$

Thus, our desired result holds for all positive integers $n, k$ and all $x, y \in \mathbb{C}$.

Summary. We give a combinatorial proof of an algebraic result of Katsuura's. Moreover, we use the proof of this result to shed some light on an interesting property of the result itself. 


\section{References}

1. A. T. Benjamin, J. J. Quinn, An alternate approach to alternating sums: A method to die for, College Math. J. 39 (2008) 191-201, http: //dx.doi.org/10.2307/27646625.

2. T. P. Dence, Some half-row sums from Pascal's triangle via Laplace transforms, College Math. J. 38 (2007) 205-209, http://dx.doi.org/10.2307/27646473.

3. D. Fengming, H. W. Kim, L. T. Yeong, A family of identities via arbitrary polynomials, College Math. J. 44 (2013) 43-47, http://dx.doi.org/10.4169/college.math.j.44.1.043.

4. H. Katsuura, Summations involving binomial coefficients, College Math. J. 40 (2009) 275-278, http: //dx . doi.org/10.4169/193113409x458723. 